\title{
EDITORIAL
}

\section{Value of the myocardial performance index in myocardial infarction}

L Galiuto

Heart 2005;91:565-567. doi: 10.1136/hrt.2004.047894

The myocardial performance index represents an easy and reproducible parameter of both systolic and diastolic left ventricular function for the risk stratification of patients following acute myocardial infarction

exclusively by AMI patients successfully treated by primary percutaneous coronary intervention (PCI); the identification of the ideal time point of MPI evaluation that correlates better with left ventricular functional outcome; and, lastly, the correlation between MPI and Doppler derived parameters of microvascular integrity.

\section{MPI AFTER SUCCESSFUL CORONARY RECANALISATION: RESULTS OF UNIFORM PATIENT POPULATION}

The role of MPI in the evaluation of post-infarct left ventricular function and of patient prognostic implications has been assessed in at least three large studies..$^{4-6}$ In all these studies, patient population was heterogeneous in terms of timing and type of recanalisation strategies, thus they included patients with necrotic damage of variable extent and differing degrees of residual left ventricular dysfunction. In these conditions, MPI values were relatively high, ranging from 0.5 in patients with lower risk, ${ }^{4}$ to 0.6 in higher risk patients, ${ }^{45}$ up to 0.8 in patients with higher mortality. ${ }^{6}$ Interestingly, Møller and colleagues ${ }^{4}$ found a significant correlation between MPI values and the risk of mortality, with values lower than 0.46 associated with a risk of 1 , values between $0.46-0.55$ with a risk of 2.1, values between $0.56-0.68$ with a risk of 2.3 , and values higher that 0.68 with a risk of 4 .

In the study by Kato and colleagues, ${ }^{3}$ average MPI values were significantly lower than those reported in previous studies, around 0.3 . This apparent discrepancy may be explained by the homogeneity of the studied population, which was comprised exclusively of patients successfully treated by primary angioplasty within six hours from the beginning of symptoms; thus necrotic damage was minor, producing only limited left ventricular dysfunction, which is likely to be associated with lower MPI values. However, the results reported by Kato and colleagues $^{3}$ have to be considered as being only preliminary, since they refer to a relatively small population and a control group of normal subjects is lacking. These data need to be confirmed in larger studies and a possible correlation between MPI values and the extent of myocardial necrosis still needs to be established.

Correspondence to: Dr Leonarda Galiuto, Institute of Cardiology, Catholic University of the Sacred Heart, Policlinico A. Gemelli, Largo A. Gemelli, 800136 Rome, Italy; lgaliuto@rm.unicatt.it identified by Tei and colleagues, ${ }^{2}$ with the of providing a comprehensive parameter of cardiac function to be used in the serial tion and risk stratification of patients with lar systolic and diastolic dysfun various pathogenesis, extent, and evolution. In this issue of Heart, Kato and colleagues ${ }^{3}$ report their experience with the use of MPI in the prediction of left ventricular functional outcome in patients with early recanalisation after anterior AMI.

Different investigators have previously reported the incremental prognostic value of MPI after AMI. However, the additional information provided by the study of Kato and colleagues ${ }^{3}$ reside in at least three aspects of the investigation: the selection of the study population made
Abbreviations: $A M I$, acute myocardial infarction; $M C E$, myocardial contrast echocardiography; $\mathrm{MPI}$, myocardial performance index; $\mathrm{PCl}$, percutaneous coronary intervention 


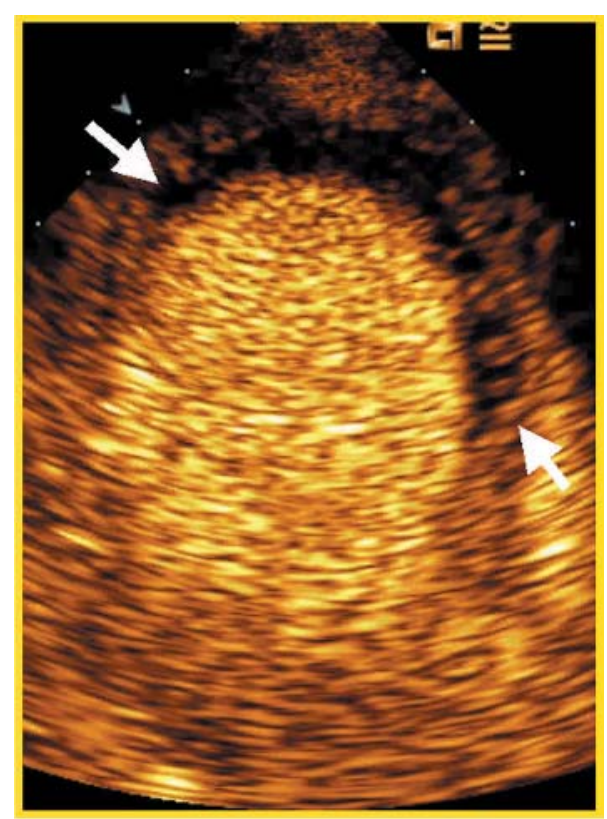

Figure 1 Myocardial contrast echocardiographic (MCE) image of a patient with acute myocardial infarction after primary coronary angioplasty. Microvascular perfusion is visualised as the orange signal within the left ventricular wall in long axis view using a novel ultrasound software, Cadence Pulse Sequencing (Sequoia, Siemens), with the continuous infusion $(5 \mathrm{ml}$ at $1 \mathrm{ml} / \mathrm{min}$ ) of Sonovue. The black apical area within arrows represents the area of post-ischaemic microvascular damage (no reflow).

\section{MPI AND POST-ISCHAEMIC MICROVASCULAR INTEGRITY}

Particularly interesting and novel is the correlation found by Kato and colleagues ${ }^{3}$ between MPI values and parameters related to post-ischaemic microvascular integrity derived from Doppler flow measurements of the infarct related coronary artery. The authors report a significant negative correlation between MPI and peak systolic coronary flow velocity, a positive correlation with coronary diastolic deceleration rate, and a negative correlation with deceleration time; furthermore, MPI values are higher in patients with systolic retrograde flow. In other words, patients with post-AMI left ventricular dysfunction, as documented by higher MPI, have a coronary flow pattern characterised by shorter coronary diastolic deceleration time with higher deceleration rate and systolic reverse flow.

In the multicentre study of Lepper and colleagues, coronary blood flow was measured by Doppler guidewire immediately after and 24 hours after PCI for AMI, and microvascular integrity was studied by MCE before and 24 hours after PCI. Compared with patients with reperfusion, patients with MCE non-reperfusion had a lower systolic flow velocity immediately after PCI and after 24 hours, more frequent early systolic retrograde flow, and a shorter diastolic deceleration time immediately after PCI and after 24 hours. Thus, coronary blood flow velocity pattern significantly correlates with microvascular damage and the occurrence of reperfusion injury.

The integration of MCE, MPI, and Doppler flow data allow us to conclude that if recanalisation of the infarct related artery is associated with microvascular damage as documented by MCE, coronary blood flow pattern is significantly impaired and left ventricular systolic and diastolic function is compromised, as demonstrated by high MPI values. In this context, MPI evaluation appears to be an easy and effective way to measure the effects of a recanalisation strategy on myocardial function, thus providing, at the same time, information on the microvascular patency status. However, a clear answer on the role of MPI in the evaluation of microvascular integrity may be given by the direct comparison between this index of systolic and diastolic left ventricular function and methods, such as MCE or magnetic resonance imaging, specifically devoted to the direct assessment of coronary microcirculation.

\section{SERIAL MPI: ROLE OF TIMING OF ASSESSMENT}

In the setting of AMI, evolving microvascular and myocardial changes occur after successful coronary recanalisation. If a significant number of microvessels are preserved anatomically intact, the re-establishment of coronary flow initially generates a hyperaemic response, followed by rapid normalisation with return to a microvascular reflow pattern. ${ }^{8}$ If microvessels are anatomically compromised by prolonged ischaemia, recanalisation of the infarct related artery does not produce significant hyperaemia and, more importantly, lack of significant reflow occurs at the microvascular level. No reflow can be accurately documented by MCE within 24 hours after percutaneous intervention. However, the no reflow phenomenon may still be dynamic and in at least $50 \%$ of cases a spontaneous and progressive recovery of microvascular perfusion occurs within the first month after AMI. ${ }^{9}$ Similarly, after coronary recanalisation, post-ischaemic myocardium undergoes progressive changes characterised by initial recovery of viable but dysfunctioning areas (stunned myocardium) and then possible adaptation of the entire left ventricle to the lack of contractile myocardium with progressive involvement of the border zone in the remodelling process. Taking all these microvascular and myocardial changes into consideration, the optimal timing to assess the extent of microvascular damage and to predict myocardial viability appears to be day 1 after coronary recanalisation. ${ }^{10}$ In complete accord with these previous findings and with the pathophysiology of microvascular and myocardial temporal changes after ischaemia-reperfusion, in the study of Kato and colleagues ${ }^{3}$ MPI on day 1 correlates better with left ventricular function and volume changes than MPI assessed immediately after recanalisation and at day 7 . These data confirm that day $\mathrm{l}$ is the best time to evaluate post-ischaemic function, thus avoiding the very early phase during which stunned myocardium is still dysfunctioning, and the later phase when, although viable myocardium is likely to have recovered its contractile state, the remodelling process has started, along with impairment of global function caused by the widespread use of $\beta$ blockers.

Similarly, MPI at day 1 shows a good correlation with Doppler parameters of coronary reflow, thus allowing the authors to conclude that this index is a reasonable indicator, not only of systolic and diastolic left ventricular function, but also of microvascular flow. Although day 1 appears to be the ideal moment to assess microvascular function, it is noteworthy that, in this study, MPI has been correlated with coronary flow data obtained immediately after infarct related artery recanalisation, when reactive hyperaemia usually leads to underestimation of final post-ischaemic microvascular damage. Future studies using non-invasive evaluation of coronary flow by transthoracic Doppler echocardiography ${ }^{11}$ might provide serial assessment of infarct related artery reflow pattern, thus allowing not only the time course of such a dynamic phenomenon to be followed, but also to compare indexes of perfusion and function obtained at the same time.

\section{REFERENCES}

1 Bolognese L, Carrabba N, Parodi G, et al. Impact of microvascular dysfunction on left ventricular remodeling and long-term clinical outcome after 
primary coronary angioplasty for acute myocardial infarction. Circulation 2004; 109:1121-6.

2 Tei C. New noninvasive index of combined systolic and diastolic ventricular function. J Cardiol 1995;26:135-6.

3 Kato M, Dote K, Sasaki S, et al. Myocardial performance index for assessment of left ventricular outcome in successfully recanalised anterior myocardial infarction. Heart 2005;91:583-8.

4 Møller JE, Egstrup K, Køber L, et al. Prognostic importance of systolic and diastolic function after acute myocardial infarction. Am Heart $J$ 2003:145:147-53.

5 Poulsen SH, Jensen SE, Nielsen JC, et al. Serial changes and prognostic implications of a Doppler-derived index of combined left ventricular systolic and diastolic myocardial performance in acute myocardial infarction. Am J Cardiol 2000;85:19-25.

6 Yeo TC, Dujardin KS, Tei C, et al. Value of a Doppler-derived index combining systolic and diastolic time intervals in predicting outcome in primary pulmonary hypertension. Am J Cardiol 1998;81:1157-61.
7 Lepper W, Sieswerda GT, Franke A, et al. Repeated assessment of coronary flow velocity pattern in patients with first acute myocardial infarction. J Am Coll Cardiol 2002; 17:1283-9.

8 Galiuto L, DeMaria AN, May-Newman K, et al. Evaluation of dynamic changes in microvascular flow during ischemia-reperfusion by myocardial contrast echocardiography. J Am Coll Cardiol 1998;32:1096-101.

9 Galiuto L, Lombardo A, Maseri A, et al. Temporal evolution and functional outcome of no-reflow: sustained and spontaneously reversible patterns following successful coronary recanalisation. Heart 2003;89:731-7.

10 Sakuma T, Otsuka M, Okimoto T, et al. Optimal time for predicting myocardial viability after successful primary angioplasty in acute myocardial infarction: a study using myocardial contrast echocardiography. Am J Cardiol 2001;87:687-92.

11 Caiati C. Montaldo C, Zedda N, et al. Validation of a new noninvasive method (contrast-enhanced transthoracic second harmonic echo Doppler) for the evaluation of coronary flow reserve: comparison with intracoronary Doppler flow wire. J Am Coll Cardiol 1999;34:1193-200.

\section{IMAGES IN CARDIOLOGY}

\section{Diagnosis of myocarditis by cardiovascular magnetic resonance}

\begin{abstract}
A
33 year old white man presented with severe, tight, central chest pain following a flu-like illness. He had no significant risk factors for premature ischaemic heart disease and there was no history of cocaine usage. A 12 lead ECG demonstrated $1 \mathrm{~mm}$ ST segment elevation in leads II, III, and $\mathrm{aVF}$, and leads V5 and V6 (upper panel). Initial creatine kinase was $3030 \mathrm{IU} / \mathrm{l}$ and troponin $\mathrm{T}$ was positive. Transthoracic echocardiography was normal and the patient was referred for cardiovascular magnetic resonance (CMR) with a provisional diagnosis of myocarditis. On cine imaging, CMR showed normal biventricular function with no focal wall thinning or wall motion abnormalities. However, late gadolinium enhancement demonstrated epicardial enhancement in the basal inferior, basal inferolateral, and basal anterolateral segments (panels A and B). This pattern of enhancement did not correspond to myocardial infarction (MI) in a coronary artery territory and supported the clinical diagnosis of myocarditis. Subsequent coronary angiography was normal. Although the one year follow up ECG showed complete resolution of the ST segments, repeat contrast enhanced CMR demonstrated persistent, but reduced, epicardial enhancement in the same basal segments.

Myocarditis can be caused by a wide variety of infectious agents and can be detected with the technique of late gadolinium enhancement. This case highlights the use of this technique for establishing a definitive diagnosis between the more common clinical entity of MI and the relatively uncommon condition of myocarditis. In addition, such unusual CMR appearances in conjunction with the appropriate clinical setting could obviate the necessity for endomyocardial biopsy in these patients.
\end{abstract}

A Varghese

$S$ Davies

D J Pennell

a.varghese@rbh.nthames.nhs.uk

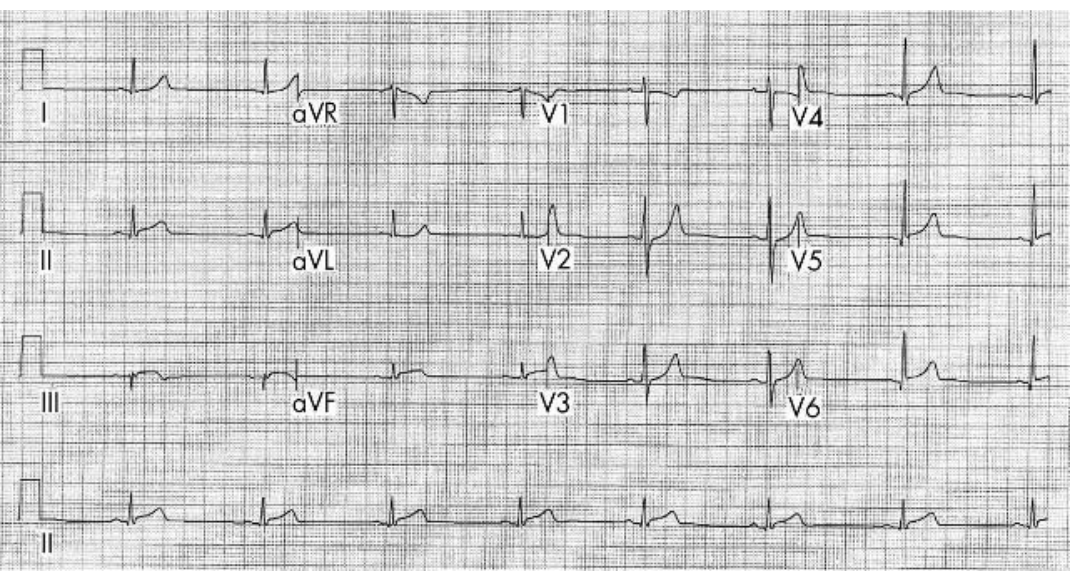

ECG from initial presentation with $1 \mathrm{~mm}$ ST segment elevation in the inferolateral leads.
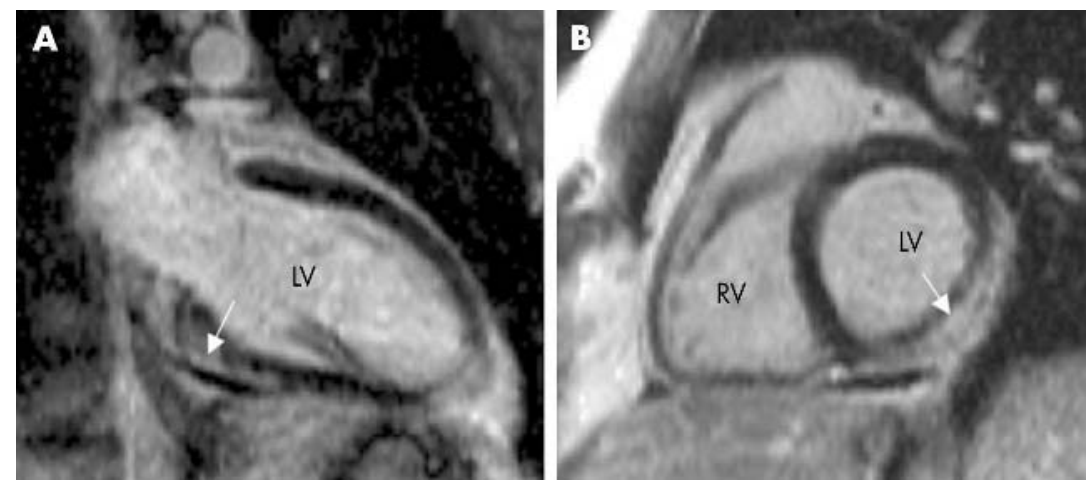

Vertical long axis (A) and basal short axis (B) views from the initial CMR showing epicardial late gadolinium enhancement in the basal inferior and basal inferolateral segments (arrows). LV, left ventricle; RV, right ventricle. 